\title{
NEW CHEMICAL PROFILES FOR THE ASTEROSEISMOLOGY OF ZZ CETI STARS
}

\author{
L. G. Althaud ${ }^{1,2}$, A. H. Córsico ${ }^{1,2}$, A. Bischoff-Kim ${ }^{3}$, A. D. Romero ${ }^{1,2}$, I. Renedo ${ }^{4,5}$, E. García-Berro ${ }^{4.5}$, \\ AND M. M. Miller Bertolami ${ }^{1,2}$ \\ ${ }^{1}$ Facultad de Ciencias Astronómicas y Geofísicas, Universidad Nacional de La Plata, Paseo del Bosque s/n. (1900) La Plata, Argentina \\ ${ }^{2}$ Instituto de Astrofísica de La Plata, IALP (CCT La Plata), CONICET-LINLP, Argentina \\ ${ }^{3}$ Department of Chemistry, Physics and Astronomy, CBX 82, Georgia College \& State University, Milledgeville, GA 31061, USA \\ ${ }^{4}$ Departament de Física Aplicada. Universitat Politècnica de Catalunya. c/Esteve Terrades 5. 08860 Castelldefels. Spain \\ ${ }^{5}$ Institute for Space Studies of Catalonia. c/Gran Capità 2-4. Edif. Nexus 104.08034 Barcelona. Spain; althaus@ fcaglp.unlp.edu.ar \\ Received 2010 March 23; accepted 2010 May 14; published 2010 June 21
}

\begin{abstract}
We compute new chemical profiles for the core and envelope of white dwarfs appropriate for pulsational studies of ZZ Ceti stars. These profiles are extracted from the complete evolution of progenitor stars, evolved through the main sequence and the thermally pulsing asymptotic giant branch (AGB) stages, and from time-dependent element diffusion during white dwarf evolution. We discuss the importance of the initial-final mass relationship for the white dwarf carbon-oxygen composition. In particular, we find that the central oxygen abundance may be underestimated by about $15 \%$ if the white dwarf mass is assumed to be the hydrogen-free core mass before the first thermal pulse. We also discuss the importance for the chemical profiles expected in the outermost layers of ZZ Ceti stars of the computation of the thermally pulsing AGB phase and of the phase in which element diffusion is relevant. We find a strong dependence of the outer layer chemical stratification on the stellar mass. In particular, in the less massive models, the double-layered structure in the helium layer built up during the thermally pulsing $\mathrm{AGB}$ phase is not removed by diffusion by the time the ZZ Ceti stage is reached. Finally, we perform adiabatic pulsation calculations and discuss the implications of our new chemical profiles for the pulsational properties of ZZ Ceti stars. We find that the whole g-mode period spectrum and the mode-trapping properties of these pulsating white dwarfs as derived from our new chemical profiles are substantially different from those based on chemical profiles widely used in existing asteroseismological studies. Thus, we expect the asteroseismological models derived from our chemical profiles to be significantly different from those found thus far.
\end{abstract}

Key words: stars: evolution - stars: interiors - stars: oscillations - white dwarfs

Online-only material: color figures

\section{INTRODUCTION}

Pulsating DA (H-rich atmospheres) white dwarfs, also called ZZ Ceti or DAV stars, are the most numerous class of degenerate pulsators, with over 143 members known today (Winget \& Kepler 2008). Since the discovery of the first ZZ Ceti star, HL Tau 76, by Landolt (1968), there has been a continuous effort to model the interior of these variable stars. ZZ Ceti stars are found within a very narrow strip of effective temperatures $\left(10,500 \mathrm{~K} \lesssim T_{\text {eff }} \lesssim 12,500 \mathrm{~K}\right)$. They are characterized by multiperiodic brightness variations of up to 0.30 mag caused by spheroidal, non-radial $g$-modes of low degree $(\ell \leqslant 2)$ with periods between 100 and $1200 \mathrm{~s}$. The driving mechanism thought to excite the pulsations near the blue edge of the instability strip is the $\kappa-\gamma$ mechanism that takes place in the hydrogen partial ionization zone (Dolez \& Vauclair 1981; Dziembowski \& Koester 1981; Winget et al. 1982). Also, the "convective driving" mechanism has been proposed-first by Brickhill (1991) and later re-examined by Goldreich \& Wu (1999). It appears to be responsible for mode driving once a thick convection zone has developed at the stellar surface. The comparison of the observed pulsation periods in white dwarfs and the periods computed for appropriate theoretical models (white dwarf asteroseismology) allows one to infer details of their origin, internal structure, and evolution (Winget \& Kepler 2008; Fontaine \& Brassard 2008). In particular, the stellar mass, the thickness of the outer envelopes, the core chemical composition, magnetic fields, and rotation rates can be determined from the observed periods. In addition, the asteroseismology of
ZZ Ceti stars is a valuable tool for studying axions (Isern et al. 1992, 2010; Córsico et al. 2001; Bischoff-Kim et al. 2008) and crystallization (Montgomery et al. 1999; Córsico et al. 2004, 2005; Metcalfe et al. 2004; Kanaan et al. 2005). Finally, the temporal changes in the observed periods can help detect planets orbiting around white dwarfs (Mullally et al. 2008).

The first published complete set of DA white dwarf models suitable for asteroseismology was that of Tassoul et al. (1990). A large parameter space was explored in such a monumental study, and for a long time (since the early 1980s) this set of models represented the state of the art in the area. The pulsation properties of these models were thoroughly explored in a series of important papers by Brassard et al. (1991, 1992a, 1992b). As important as these models were at that time, they suffer from a number of shortcomings. For instance, the core of the models is made of pure carbon, while stellar evolution calculations indicate that cores of typical white dwarfs are made of a mixture of carbon and oxygen. Also, the carbon/helium $(\mathrm{C} / \mathrm{He})$ and helium/hydrogen $(\mathrm{He} / \mathrm{H})$ chemical interfaces are modeled on the basis of the assumption of the diffusive equilibrium in the "trace element approximation," an approach that involves a quasi-discontinuity in the chemical profiles at the transition regions which, in turn, leads to peaked features in the Brunt-Väisălä frequency and exaggerated modetrapping effects (Córsico et al. 2002a, 2002b). These models were employed for asteroseismological inferences of the DAVs G 226-29 (Fontaine et al. 1992) and GD 154 (Pfeiffer et al. 1996). More recently, Pech et al. (2006) and Pech \& Vauclair (2006) have presented asteroseismological analysis on HL Tau 
76 and G 185-32, respectively, by employing similar DA white dwarf models, although with updated input physics.

The models of Bradley (1996) constituted a substantial improvement in the field. These models have carbon-oxygen cores in varying proportions, and the $\mathrm{C} / \mathrm{He}$ and $\mathrm{He} / \mathrm{H}$ chemical interfaces are more realistic. Perhaps the most severe shortcoming of these models is the (unrealistic) ramp-like shape of the core carbon-oxygen chemical profiles. These DA models were the basis of the very important asteroseismological studies on the DAVs G 29-38 (Bradley \& Kleinman 1997), G 117-B15A and R 548 (Bradley 1998), GD 165 and L 19-2 (Bradley 2001), and G 185-32 (Bradley 2006).

The next step in improving the modeling of DAVs was given by Córsico et al. (2002a) and Benvenuto et al. (2002a), who employed evolutionary models characterized by $\mathrm{He} / \mathrm{H}$ chemi$\mathrm{cal}$ interfaces resulting from a time-dependent element diffusion treatment (Althaus \& Benvenuto 2000), and the carbon-oxygen core chemical structure extracted from the evolutionary computations of Salaris et al. (1997). The use of very smooth outer chemical interfaces, as shaped by chemical diffusion, revealed that the use of the trace element approximation turns out to be inappropriate for modeling the shape of the chemical interfaces in a DA white dwarf. This grid of models was employed in an asteroseismological study of G 117-B15A (Benvenuto et al. 2002a). For these sequences, the starting configurations for the white dwarf evolution were obtained through an artificial procedure, and not as result of evolutionary computations of the progenitor stars.

Recently, Castanheira \& Kepler (2008, 2009) have carried out an extensive asteroseismological study of DAVs by employing DA white dwarf models similar to those of Bradley (1996), but with a simplified treatment of the core chemical structure, by somewhat arbitrarily fixing the central abundances to $50 \%$ oxygen and 50\% carbon. The $\mathrm{He} / \mathrm{H}$ chemical interfaces adopted for these models are a parameterization of the realistic chemical profiles resulting from time-dependent element diffusion (Althaus et al. 2003). The study includes the "classical" DAVs and also the recently discovered SDSS DAVs. In total, $83 \mathrm{ZZ}$ Ceti stars are analyzed. An important result of these studies is that the thickness of the $H$ envelopes inferred from asteroseismology is in the range $10^{-4} \geq M_{\mathrm{H}} / M_{*} \gtrsim 10^{-10}$. with a mean value of $M_{\mathrm{H}} / M=5 \times 10^{-7}$. This suggests that an important fraction of DAs characterized by envelopes substantially thinner than predicted by the standard evolution theory could exist, with the consequent important implications for the theory of white dwarf formation. However, these results do not include the possible effects of realistic carbon-oxygen profiles on the asteroseismological fits.

Almost simultaneously with the study of Castanheira \& Kepler (2008, 2009), Bischoff-Kim et al. (2008) performed a new asteroseismological study on G 117-B15A and R 548 by employing DA white dwarf models similar to those employed by Castanheira \& Kepler (2008, 2009), but incorporating realistic core chemical profiles according to Salaris et al. (1997). The results of this work are in reasonable agreement with previous studies on these $\mathrm{ZZ}$ Ceti stars. However, the mass and effective temperatures found by Bischoff-Kim et al. (2008) for G 117-B15A are rather high (especially the mass, at $0.66 M_{\odot}$ ). Recently, Bognár et al. (2009) have employed the same asteroseismological modeling to study the pulsations of the ZZ Ceti star KUV 02464+3239. Finally, Bischoff-Kim (2009) presented the results of an asteroseismological analysis of two DAVs with rich pulsation spectrum, G $38-29$ and R 808 based on simi- lar models, with parameterized, smooth ramp-like core profiles. These models are able to reproduce the observed period spectra reasonably well, though some assumptions about the $m$ and $\ell$ identification of modes were made.

White dwarf stellar models with realistic chemical profiles are crucial to correctly assess the adiabatic period spectrum and mode-trapping properties of the DAVs, which lies at the core of white dwarf asteroseismology (Brassard et al. 1992a; Bradley 1996; Córsico et al. 2002b). In this paper, we compute for the first time consistent chemical profiles for both the core and the envelope of white dwarfs with various stellar masses appropriate for detailed asteroseismological fits of $\mathrm{ZZ}$ Ceti stars. These chemical profiles are extracted from the full and complete evolution of progenitor stars from the zero age main sequence (ZAMS), to the thermally pulsing and massloss phases on the asymptotic giant branch (AGB), and from time-dependent element diffusion predictions during the white dwarf stage. ${ }^{6}$ These profiles will be valuable in conducting future asteroseismological studies of ZZ Ceti stars that intend to include realistic chemical profiles throughout the interior of white dwarfs. To assess the impact of these new profiles on the theoretical pulsational inferences, we perform adiabatic pulsation computations and compare the resulting periods with the pulsational inferences based on the most widely used chemical profiles in existing asteroseismological fits.

This paper is organized as follows. In Section 2, we provide a description of the input physics assumed in the evolutionary calculations of relevance for the chemical composition. In Section 3, we discuss the importance of the initial-final mass relationship for the expected white dwarf carbon-oxygen composition. The resulting chemical profiles are described in detail in Section 4. The implications of our new chemical profiles for the pulsational properties of ZZ Ceti stars are discussed in Section 5. We conclude in Section 6 by summarizing our findings.

\section{INPUT PHYSICS}

The chemical profiles presented in this paper have been extracted from full and complete evolutionary calculations for both the white dwarfs and their progenitor stars, by using an updated version of the LPCODE stellar evolutionary code-see Althaus et al. (2005) and references therein. This code has recently been employed to study different aspects of the evolution of low-mass stars, such as the formation and evolution of DA white dwarfs (Renedo et al. 2010), H-deficient white dwarfs, PG 1159, and extreme horizontal branch stars (Althaus et al. 2005, 2009a; Miller Bertolami \& Althaus 2006; Miller Bertolami et al. 2008), as well as the evolution of He-core white dwarfs with high-metallicity progenitors (Althaus et al. 2009b). It has also been used to study the initial-final mass relation in Salaris et al. (2009), where a test and comparison of LPCODE with other evolutionary codes has also been made. Details of LPCODE can be found in these works. In what follows, we comment on the main input physics that is relevant for his work. We assume the metallicity of progenitor stars to be $Z=0.01$.

The LPCODE evolutionary code considers a simultaneous treatment of non-instantaneous mixing (and extra-mixing if present) and burning of elements (Althaus et al. 2003). The nuclear network accounts explicitly for the following elements: ${ }^{1} \mathrm{H},{ }^{2} \mathrm{H},{ }^{3} \mathrm{He},{ }^{4} \mathrm{He},{ }^{7} \mathrm{Li},{ }^{7} \mathrm{Be},{ }^{12} \mathrm{C},{ }^{13} \mathrm{C},{ }^{14} \mathrm{~N},{ }^{15} \mathrm{~N},{ }^{16} \mathrm{O},{ }^{17} \mathrm{O},{ }^{18} \mathrm{O},{ }^{19} \mathrm{~F}$, ${ }^{211} \mathrm{Ne}$, and ${ }^{22} \mathrm{Ne}$, together with 34 thermonuclear reaction rates

\footnotetext{
6 The chemical profiles for the core and envelope of our models appropriate
} for ZZ Ceti stars are available at http://www.fcaglp.unlp.edu.ar/evolgroup. 
of the $p p$-chains, $\mathrm{CNO}$ bi-cycle, helium burning, and carbon ignition that are identical to those described in Althaus et al. (2005), with the exception of ${ }^{12} \mathrm{C}+p \rightarrow{ }^{13} \mathrm{~N}+\gamma \rightarrow{ }^{13} \mathrm{C}+$ $e^{+}+\nu_{\mathrm{e}}$ and ${ }^{13} \mathrm{C}(p, \gamma){ }^{14} \mathrm{~N}$, which were taken from Angulo et al. (1999). The ${ }^{12} \mathrm{C}(\alpha, \gamma){ }^{16} \mathrm{O}$ reaction rate, of special relevance for the carbon-oxygen stratification of the emerging white dwarf, was taken from Angulo et al. (1999). The standard mixing length theory for convection - with the free parameter $\alpha=1.61$ - has been adopted. With this value, the present luminosity and effective temperature of the Sun, at an age of $4570 \mathrm{Myr}$, are reproduced by LPCODE when $Z=0.0164$ and $X=0.714$ are adopted-in agreement with the $Z / X$ value of Grevesse \& Sauval (1998).

Except for the evolutionary stages corresponding to the thermally pulsing AGB phase, we have considered the occurrence of extra-mixing episodes beyond each convective boundary following the prescription of Herwig et al. (1997). We treated extra-mixing as a diffusion process by assuming that mixing velocities decay exponentially beyond each convective boundary. Specifically, we assume a diffusion coefficient given by $D_{\mathrm{OV}}=D_{\mathrm{O}} \exp \left(-2 z / f H_{\mathrm{P}}\right)$, where $H_{\mathrm{P}}$ is the pressure scale height at the convective boundary, $D_{\mathrm{O}}$ is the diffusion coefficient of unstable regions close to the convective boundary, and $z$ is the geometric distance from the edge of the convective boundary (Herwig et al. 1997). We adopted $f=0.016$ in all our sequences, a value inferred from the width of the upper main sequence. The occurrence of extra-mixing episodes during core helium burning largely determines the final chemical composition of the white dwarf core. In this sense, our treatment of time-dependent extra-mixing episodes predicts a core chemical stratification similar to that predicted by the phenomenon of "semiconvection" during central helium burning, which naturally yields the growth of the convective core (Straniero et al. 2003). Finally, the breathing pulse instability occurring toward the end of core helium burning was suppressed-see Straniero et al. (2003) for a discussion of this point. In our simulations, breathing pulses have been suppressed by gradually decreasing the parameter $f$ from the moment the helium convective core starts to recede (which occurs once the helium abundance in the core decreases below $\approx 0.05-0.1$ ). At this stage, the gradual suppression of extra-mixing toward the end of core helium burning bears no consequences for the final chemical stratification.

Other physical ingredients considered in LPCODE are the radiative opacities from the OPAL project (Iglesias \& Rogers 1996), including carbon- and oxygen-rich composition, supplemented at low temperatures with the molecular opacities of Alexander \& Ferguson (1994). During the white dwarf regime, the metal mass fraction $Z$ in the envelope is not assumed to be fixed. Instead, it is specified consistently according to the prediction of element diffusion. To account for this, we have considered radiative opacities tables from OPAL for arbitrary metallicities. For effective temperatures less than $10,000 \mathrm{~K}$ we have included the effects of molecular opacity by assuming pure hydrogen composition from the computations of Marigo $\&$ Aringer (2009). This assumption is justified because element diffusion leads to pure hydrogen envelopes in cool white dwarfs. The conductive opacities are those of Cassisi et al. (2007), and the neutrino emission rates are taken from Itoh et al. (1996) and Haft et al. (1994). For the high-density regime characteristics of white dwarfs, we have used the equation of state of Segretain et al. (1994), which accounts for all of the important contributions for both the liquid and solid phases-see Althaus et al. (2007) and references therein.
Table 1

Initial and Final Stellar Mass (in Solar Units), and the Central Oxygen Abundance (Mass Fraction) Left After Core Helium Burning, and After Rayleigh-Taylor Rehomogenization

\begin{tabular}{lccr}
\hline \hline$M_{\mathrm{ZAMS}}$ & $M_{\mathrm{WD}}$ & $X_{\mathrm{O}}(\mathrm{CHB})$ & $X_{\mathrm{O}}(\mathrm{RT})$ \\
\hline 1.00 & 0.5249 & 0.702 & 0.788 \\
1.50 & 0.5701 & 0.680 & 0.686 \\
1.75 & 0.5932 & 0.699 & 0.704 \\
2.00 & 0.6096 & 0.716 & 0.723 \\
2.25 & 0.6323 & 0.747 & 0.755 \\
2.50 & 0.6598 & 0.722 & 0.730 \\
3.00 & 0.7051 & 0.658 & 0.661 \\
3.50 & 0.7670 & 0.649 & 0.655 \\
4.00 & 0.8373 & 0.635 & 0.641 \\
5.00 & 0.8779 & 0.615 & 0.620 \\
\hline
\end{tabular}

Note. The progenitor metallicity is $Z=0.01$.

In this study, we have considered the distinct physical processes that are responsible for changes in the chemical abundance distribution during white dwarf evolution. In particular, element diffusion strongly modifies the chemical composition profile throughout their outer layers. As a result of diffusion processes, our sequences developed pure hydrogen envelopes, the thickness of which gradually increases as evolution proceeds. We have considered gravitational settling as well as thermal and chemical diffusion-but not radiative levitation, which is relevant only for the hottest and brightest post-AGB and early white dwarf cooling stages for determining the surface composition - of ${ }^{1} \mathrm{H},{ }^{3} \mathrm{He},{ }^{4} \mathrm{He},{ }^{12} \mathrm{C},{ }^{13} \mathrm{C},{ }^{14} \mathrm{~N}$, and ${ }^{16} \mathrm{O}$; see Althaus et al. (2003) for details. Our treatment of time-dependent diffusion is based on the multicomponent gas treatment presented in Burgers (1969). In LPCODE, diffusion becomes operative once the wind limit is reached at high effective temperatures (Unglaub $\&$ Bues 2000). In addition, abundance changes resulting from residual nuclear burning-mostly during the hot stages of white dwarf evolution-have been taken into account in our simulations. Finally, we considered the chemical rehomogenization of the inner carbon-oxygen profile induced by Rayleigh-Taylor (RT) instabilities following Salaris et al. (1997). These instabilities arise because of the positive molecular weight gradients that remain above the flat chemical profile left by convection during core helium burning.

\section{THE IMPORTANCE OF THE INITIAL-FINAL MASS RELATIONSHIP}

As mentioned, chemical profiles appropriate for DA white dwarfs have been derived from the full evolutionary calculations of progenitor stars for solar metallicity. To this end, the complete evolution of 10 evolutionary sequences with initial stellar mass ranging from 1 to $5 M_{\odot}$ was computed from the ZAMS through the thermally pulsing and mass-loss phases on the AGB and finally to the domain of planetary nebulae. In Table 1 , we list the stellar masses of the resulting white dwarfs, together with the initial masses of the progenitor stars on the ZAMS. Also listed in Table 1 is the central oxygen abundance both at the end of core He burning and after chemical rehomogenization by RT instabilities.

We mention that extra-mixing episodes were disregarded during the thermally pulsing AGB phase. In particular, a strong reduction (a value of $f$ much smaller than 0.016 ) of extra-mixing episodes at the base of the pulse-driven convection zone is supported by simulations of the $s$-process abundance patterns 

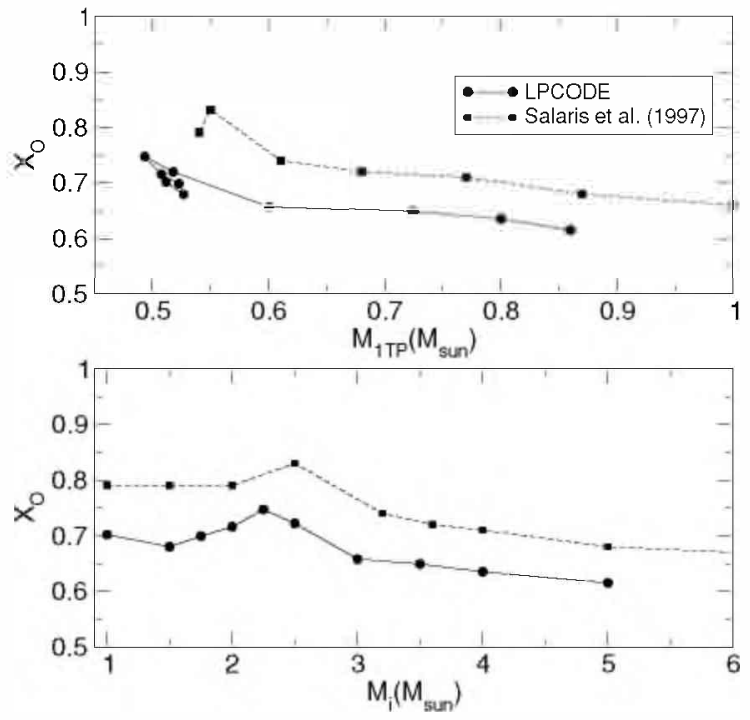

Figure 1. Central oxygen abundance (mass fraction) left after core helium burning in terms of both the hydrogen-free core mass right before the first thermal pulse (upper panel) and the initial stellar mass on the ZAMS (lower panel). The solid lines show our results while the dashed lines show those of Salaris et al. (1997).

(Lugaro et al. 2003) and, more recently, by observational inferences of the initial-final mass relation (Salaris et al. 2009). As a result, it is expected that the mass of the hydrogen-free core of our sequences gradually grows as evolution proceeds through the thermally pulsing AGB. This is because a strong reduction or suppression of extra-mixing at the base of the pulse-driven convection zone strongly inhibits the occurrence of third dredgeup, thus favoring the growth of the hydrogen-free core. We considered mass-loss episodes during the core helium burning stage and on the red giant branch following Schroder \& Cuntz (2005). During the AGB and thermally pulsing AGB phases, we adopted the maximum mass-loss rate between the prescription of Schroder \& Cuntz (2005) and that of Vassiliadis \& Wood (1993). In the case of a strong reduction of third dredge-up, as occurred in our sequences, mass loss plays a major role in determining the final mass of the hydrogen-free core at the end of the TP-AGB evolution, and thus the initial-final mass relation (Weiss \& Ferguson 2009).

We begin by examining Figure 1 which displays the central oxygen abundance by mass fraction left after core helium burning. The upper panel shows the predicted central oxygen abundance in terms of the hydrogen-free core mass right before the first thermal pulse, while the lower panel shows this quantity in terms of the initial stellar mass on the ZAMS. The predictions of our calculations-solid lines-are compared with those of Salaris et al. (1997)—dashed lines. Note the qualitatively good agreement between both sets of calculations. We recall that the final carbon-oxygen stratification of the emerging white dwarf depends on both the efficiency of the ${ }^{12} \mathrm{C}(\alpha, \gamma){ }^{16} \mathrm{O}$ reaction rate and the occurrence of extra-mixing episodes toward the late stage of core helium burning. In particular, the systematically lower oxygen abundances of our models are due mostly to our use of the cross section for the ${ }^{12} \mathrm{C}(\alpha, \gamma){ }^{16} \mathrm{O}$ reaction rate given by Angulo et al. (1999), which is smaller than the rate of Caughlan et al (1985) adopted by Salaris et al. (1997). Note that both sets of calculations predict a maximum in the central oxygen abundance at an initial mass of $M \approx 2.5 M_{\circ}$.

A careful computation of the evolutionary stages during the thermally pulsing AGB and the resulting initial-final

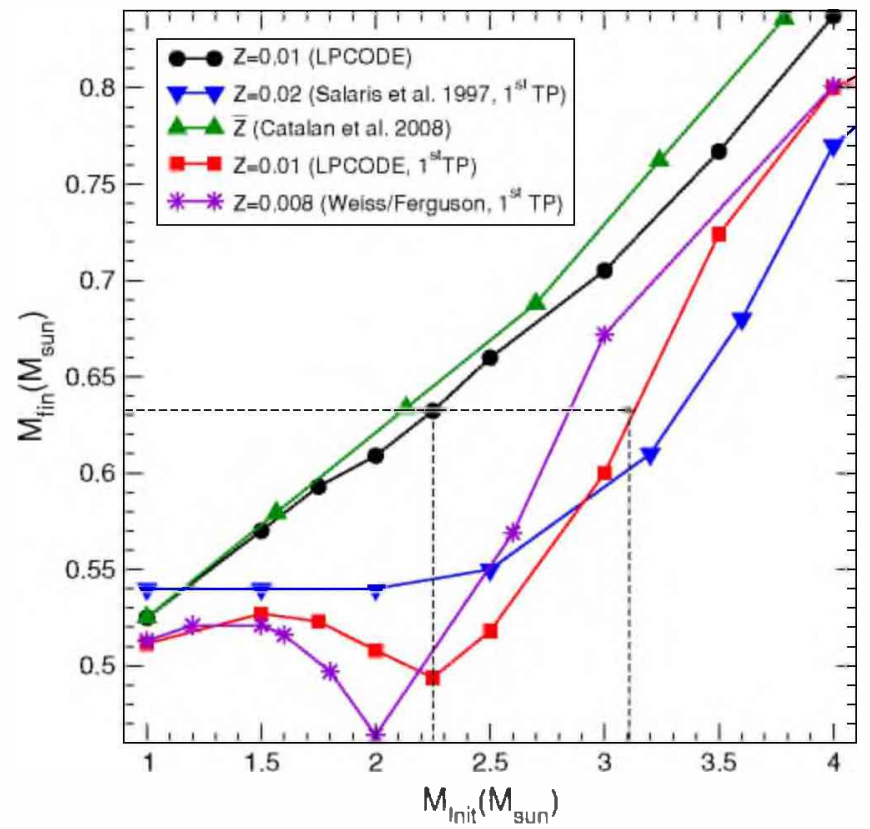

Figure 2. Initial-final mass relationships: the final mass given by the hydrogenfree core mass is depicted in terms of the initial mass of the progenitor star. In addition to the observational data from open clusters (Catalán et al. 2008), upward triangles, we show the theoretical predictions given by our calculations at the end of the TP-AGB phase and before the first thermal pulse (circles and squares, respectively). Also shown are the predictions of Weiss \& Ferguson (2009) and Salaris et al. (1997) before the first thermal pulse (stars and downward triangles, respectively).

(A color version of this figure is available in the online journal.)

mass relationship is an important aspect concerning the final carbon-oxygen composition of the white dwarf core. This can be seen by inspecting Figure 2, where various theoretical initial-final mass relationships, giving the mass of the hydrogenfree core in terms of the ZAMS mass of the progenitor, are plotted. The results shown in this figure include the predictions of our full evolutionary calculations at the end of the thermally pulsing AGB phase and at the beginning of the first thermal pulse (circles and squares, respectively). Our relationships are compared with those of Salaris et al. (1997) and Weiss \& Fergusson (2009), both at the beginning of the first thermal pulse. We also show the semiempirical initial-final mass relationship of Catalán et al. (2008), based on white dwarfs in open clusters and in common proper motions pairs with metallicities close to $Z=0.01$, the metallicity we assume for the progenitor stars of our sequences. In view of the discussion of the preceding paragraph, note the increase in the mass of the hydrogen-free core during the thermally pulsing AGB stage. As a result, the initial-final mass relationship by the end of the thermally pulsing AGB becomes markedly different from that determined by the mass of the hydrogen-free core before the first thermal pulse. For the carbon-oxygen composition expected in a white dwarf, this is an important issue. Indeed, if the mass of the hydrogenfree core is assumed to be essentially the mass of the resulting white dwarf, it is clear that a white dwarf with a given mass may correspond to different progenitor stellar masses depending on the assumed initial-final mass relationship. For instance, from our theoretical initial-to-final mass relationships, a white dwarf with $0.63 M_{\odot}$ would correspond to a progenitor star with a stellar mass of $2.25 M_{\odot}$ if the initial-final mass relationship is assessed at an advanced stage in the thermally pulsing AGB phase, or $3 M_{\odot}$ if the white dwarf mass is assumed to be the 


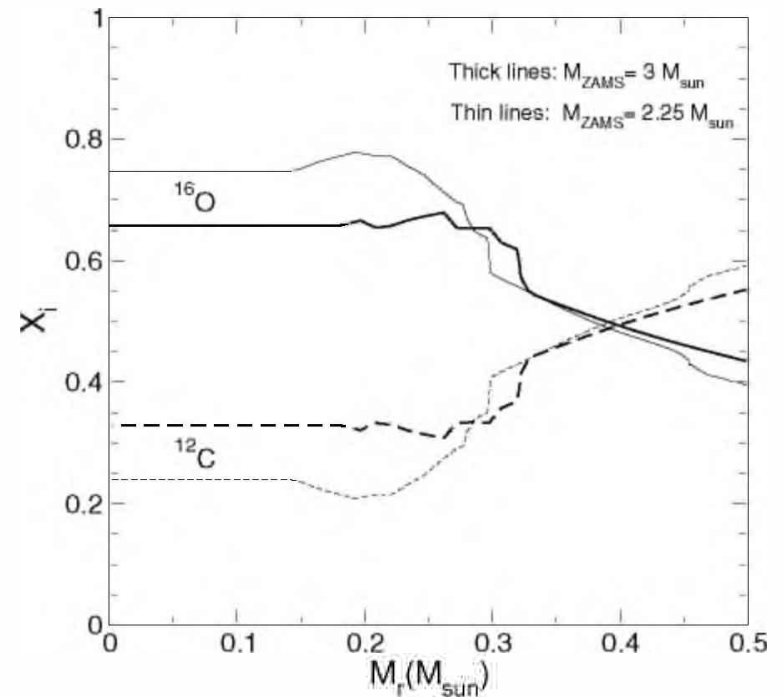

Figure 3. Inner carbon and oxygen abundance by mass for the $0.63 M_{\odot}$ white dwarf resulting from two different progenitors that lead to the same white dwarf mass. The chemical profiles correspond to progenitor stars with initial stellar masses of 3 and $2.25 M_{\odot}$ (thick and thin lines, respectively), and at stages before chemical rehomogenization by RT instability.

hydrogen-deficient core mass right before the first thermal pulse. In particular, we note that Salaris et al. (1997) adopt the mass of the hydrogen-free core at the start of the first thermal pulse as the mass of the resulting white dwarf.

The implication of this dichotomy in the mass of the progenitor star for the white dwarf carbon-oxygen composition is illustrated in Figure 3. We show the inner carbon and oxygen abundance distribution for the $0.63 M_{\odot}$ white dwarf resulting from the two different progenitors discussed previously. For illustrative purposes, chemical rehomogenization by RT instabilities has not been considered in this particular example. The thick lines display the chemical profile for the progenitor star with $3 M_{\odot}$ characterized before the first thermal pulse by a hydrogen-free core of $0.63 M_{\odot}$. The thin lines show the chemical profile for the $2.25 M_{\circ}$ progenitor which leads to the same white dwarf mass but after evolution has proceeded through the thermally pulsing AGB phase (see Figure 2). Note the different chemical profiles expected in both cases. In particular, the central oxygen abundance may be underestimated by about $15 \%$ should the white dwarf mass be assumed to be the hydrogen-free core mass right before the first thermal pulse. Note, however, that this variation is an upper limit, and it would be less for other white dwarf masses. Clearly, the initial-final mass relationship is an aspect that has to be considered in the problem of the carbon-oxygen composition expected in a white dwarf, as well as in attempts at constraining, from pulsational inferences of variable white dwarfs, the mixing processes and the efficiency of the ${ }^{12} \mathrm{C}(\alpha, \gamma){ }^{16} \mathrm{O}$ reaction rate in the core of helium burning stars. Also, it is important to realize that a larger oxygen abundance increases the cooling rate of the white dwarf because of the lower heat capacity and because an initial larger oxygen abundance reduces the energy release by phase separation on crystallization (Isern et al. 2000).

\section{THE INTERNAL CHEMICAL PROFILES}

The carbon-oxygen stratification for some selected models is shown in Figures 3 and 4. The shape of the chemical profiles before rehomogenization is given in Figure 3. Easily recogniz-

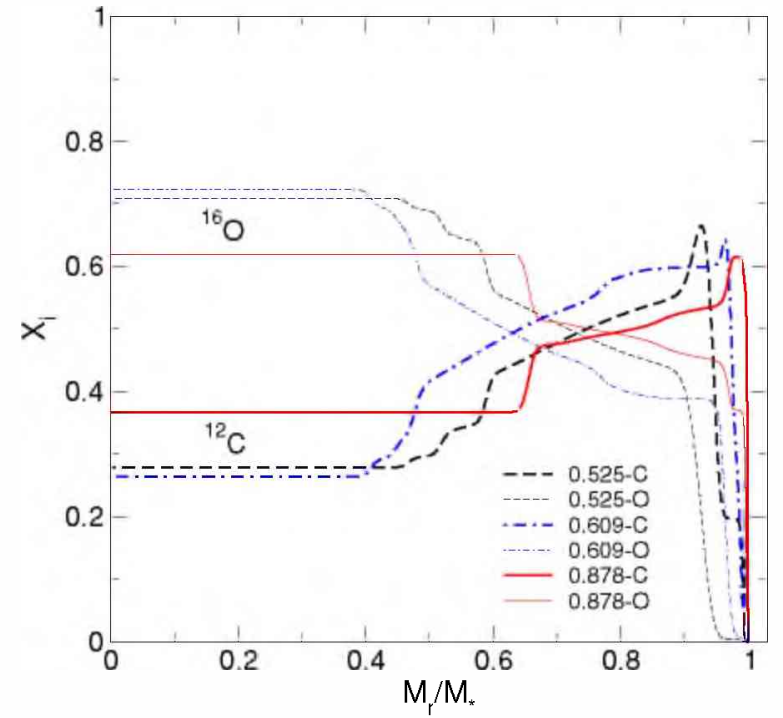

Figure 4. Inner carbon and oxygen abundance by mass for the $0.525,0.609$, and $0.878 M_{\odot}$ white dwarf models at $T_{\text {eff }} \approx 12,000 \mathrm{~K}$, and after chemical rehomogenization by RT instabilities.

(A color version of this figure is available in the online journal.)

able are the flat chemical profiles in the inner part of the core left by convection during the core helium burning, the bumps resulting from the inclusion of extra-mixing episodes beyond the fully convective core, and the signatures of the outwardmoving helium-burning shell after the end of core helium burning. Because of the larger temperatures in the heliumburning shell, the oxygen abundance decreases in the outer regions of the carbon-oxygen core.

The expected chemical profiles of some of our white dwarf models when evolution has proceeded to the domain of the $\mathrm{ZZ}$ Ceti stars are displayed in Figure 4. At this stage, chemical rehomogenization by RT instabilities has already smeared out the bumps in the inner profiles, leading to quite extended flat chemical profiles. Note the dependence of both the core chemical abundances and the location of the chemical transitions on the stellar mass. Pulsation periods in white dwarf models are very sensitive to the shapes and locations of the chemical transitions zones (see Section 5). This emphasizes the need for a detailed knowledge of the progenitor history for a realistic treatment of white dwarf evolution and pulsations. The chemical profiles in the outermost regions, resulting from prior mixing and burning events during the thermally pulsing AGB phase, are markedly modified by the diffusion processes acting during white dwarf evolution, particularly in the case of more massive models, where chemical diffusion and gravitational settling are notably more efficient. This can be better appreciated in Figure 5, where the chemical abundance distribution for white dwarf models of different stellar masses is depicted in terms of the outer mass fraction. These plots put special emphasis in the outer regions of the model. In this figure and for each stellar mass, thin lines show the chemical abundance distribution at early stages of white dwarf evolution when diffusion has not had time to act. The signature of the evolution through the thermally pulsing AGB stage on the chemical profile, particularly the formation of the helium-rich buffer and the underlying intershell region rich in helium and carbon-built up during the mixing episode at the last AGB thermal pulse-are easily visible. The presence of carbon in the intershell region stems from the shortlived convective mixing that has driven the carbon-rich zone 


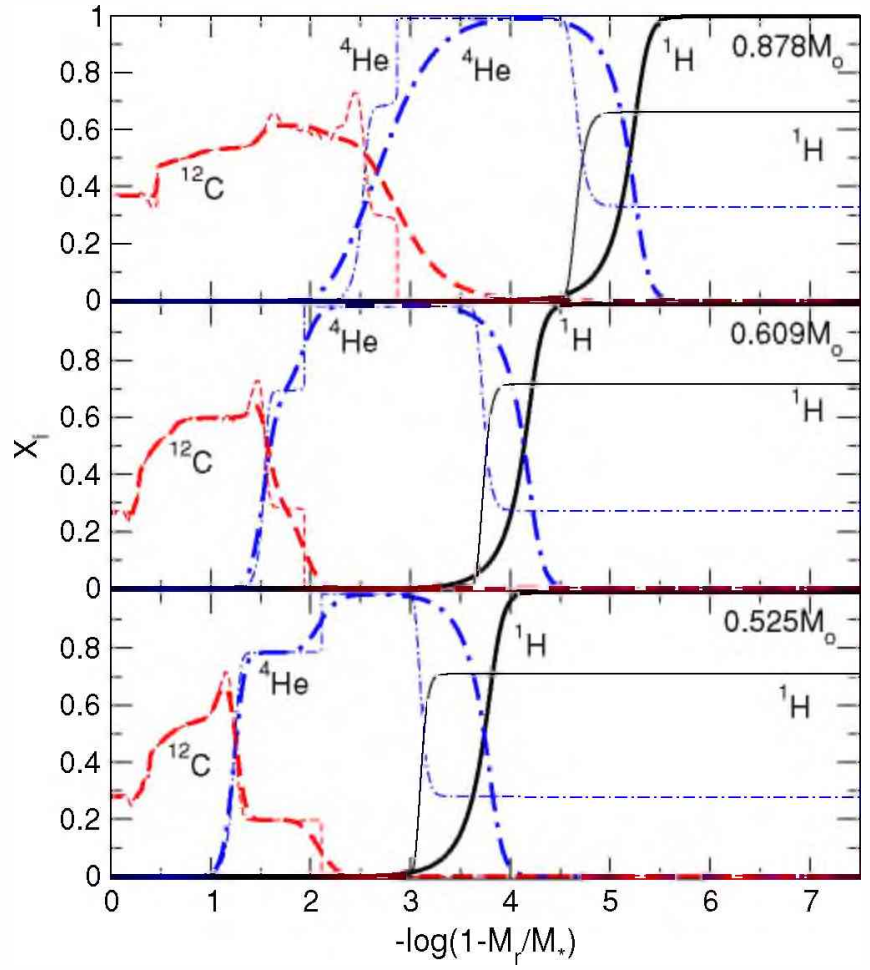

Figure 5. Abundance distribution of hydrogen, helium, and carbon in terms of the outer mass fraction for the $0.878,0.609$, and $0.525 M_{\odot}$ white dwarf models at two selected stages just after the maximum effective temperature point and near the beginning of the $Z Z$ Ceti regime (thin and thick lines, respectively).

(A color version of this figure is available in the online journal.)

upward during the peak of the last helium pulse on the AGB. Thick lines depict the situation at advanced stages, near the ZZ Ceti instability strip, when element diffusion has strongly modified the chemical abundance distribution and resulted in the formation of a thick pure hydrogen envelope plus an extended inner tail. In the more massive models, chemical diffusion leads to a significant amount of carbon in the helium buffer zone. Also near-discontinuities in the initial abundance distribution are smoothed out considerably by diffusion.

An important fact to note in Figure 5 is the dependence on the stellar mass of the outer layer chemical stratification expected in ZZ Ceti stars. Indeed, for the more massive models, diffusion has strongly modified the chemical abundance distribution, eroding the intershell region by the time evolution has reached the domain of the ZZ Ceti instability strip. This is in contrast with the situation encountered in our less massive models, where the intershell region is not removed by diffusion. This is because element diffusion is less efficient in less massive models and also because the intershell is thicker, with the subsequent longer diffusion timescales. Regarding white dwarf asteroseismology, these are not minor issues, since the presence of a double-layered structure in the helium-rich layers is expected to affect the theoretical $g$-mode period spectrum of ZZ Ceti stars, as it does for pulsating DB (He-rich) white dwarf stars (Metcalfe et al. 2003). However, we mention that the thickness of the intershell region also depends somewhat on the number of thermal pulses during the $\mathrm{AGB}$ experienced by the progenitor star.

Finally, we have explored whether the shape of the $\mathrm{He} / \mathrm{H}$ chemical interface has a dependence on the thickness of the $\mathrm{H}$ envelope. This is an important issue, because predictions of the exact value of the $H$ envelope mass are tied to the precise mass loss history along the previous $A G B$ and postAGB phase, and particularly to the occurrence of late thermal pulses. We have generated additional, artificial sequences with $\mathrm{H}$ envelopes much thinner than those of our models. These sequences were created at high luminosities from a hot model with $M$. $=0.609 M_{\odot}$. This artificial procedure took place at luminosities high enough as to ensure that the models become physically sound at stages far before the domain of the ZZ Ceti stars. We found that diffusion rapidly leads to pure hydrogen envelopes, but the shape of the $\mathrm{He} / \mathrm{H}$ chemical interfaces-by the time evolution has proceeded to the ZZ Ceti stage - is almost the same independently of the thickness of the H envelope.

\section{PULSATION PROPERTIES: COMPARISON WITH PREVIOUS CALCULATIONS}

White dwarf asteroseismology is sensitive to the precise shape of the internal chemical profiles. The entire $g$-mode period spectrum and mode-trapping properties of pulsating white dwarfs are very sensitive to the fine details of the chemical profiles of both the core and the envelope of the star. This extreme sensitivity has been exploited with some success in several pulsation studies to infer the core chemical structure-e.g., Metcalfe (2003) for the case of pulsating DB white dwarfs-and the thickness of the He and H envelopes-e.g., Bradley $(1998,2001)$ for DAV stars.

In this section, we perform a comparison between the pulsation properties derived from our new chemical profiles and those based on the most widely used chemical profiles in existing asteroseismological studies. To assess the adiabatic pulsation properties of our white dwarf models we employ the numerical code described in Córsico \& Althaus (2006). We refer the reader to that paper for details. With the aim of simplifying our analysis, we elect a template DA white dwarf model with $M_{*}=0.6096 M_{\bigcirc}, T_{\text {eff }} \sim 12,000 \mathrm{~K}$, and a thick hydrogen envelope $\left(M_{\mathrm{H}} \sim 10^{-4} M\right.$, ). This is a canonical model of a DAV star with an average mass located in the middle of the observed $Z Z$ Ceti instability strip. In Figure 6, we depict the internal chemical profile (upper panel), the Ledoux term (middle panel), and the logarithm of the squared Brunt-Väisălä and Lamb frequencies (lower panel) for dipole modes corresponding to our template model. The Ledoux term and the Brunt-Vảisalá frequency are computed as in Córsico \& Althaus (2006). Our model is characterized by three chemical transition regions: a double chemical interface of oxygen and carbon located at the core region $(0.2 \lesssim-\log q \lesssim 0.8)$, a triple chemical interface of oxygen, carbon, and helium located at $1.2 \lesssim-\log q \lesssim 2.3$, and finally, a double chemical interface of $\mathrm{He} / \mathrm{H}$ located at $3.0 \leqq-\log q \lesssim 4.5$. The core chemical profile is typical of situations in which extra-mixing episodes beyond the fully convective core (like overshooting) during the core He burning phase are allowed to operate. The smoothness of the $\mathrm{He} / \mathrm{H}$ chemical interface, on the other hand, is the result of the time-dependent element diffusion processes. The existence of these three chemical interfaces induces the "bumps" in the profile of the Brunt-Vaisälä frequency. The number of these bumps, as well as their heights and widths, strongly affect the whole structure of the pulsation spectrum of the star.

In Figure 7, we show the situation in which our template model is characterized by a ramp-like core chemical structure of the kind used by Bradley $(1996,1998,2001)$ and more recently by Bischoff-Kim (2009). In this case, the core chemical profile is not the result of stellar evolution calculations, but parameterized. These kinds of chemical profiles have been widely employed in asteroseismology of white dwarfs because they allow a full 

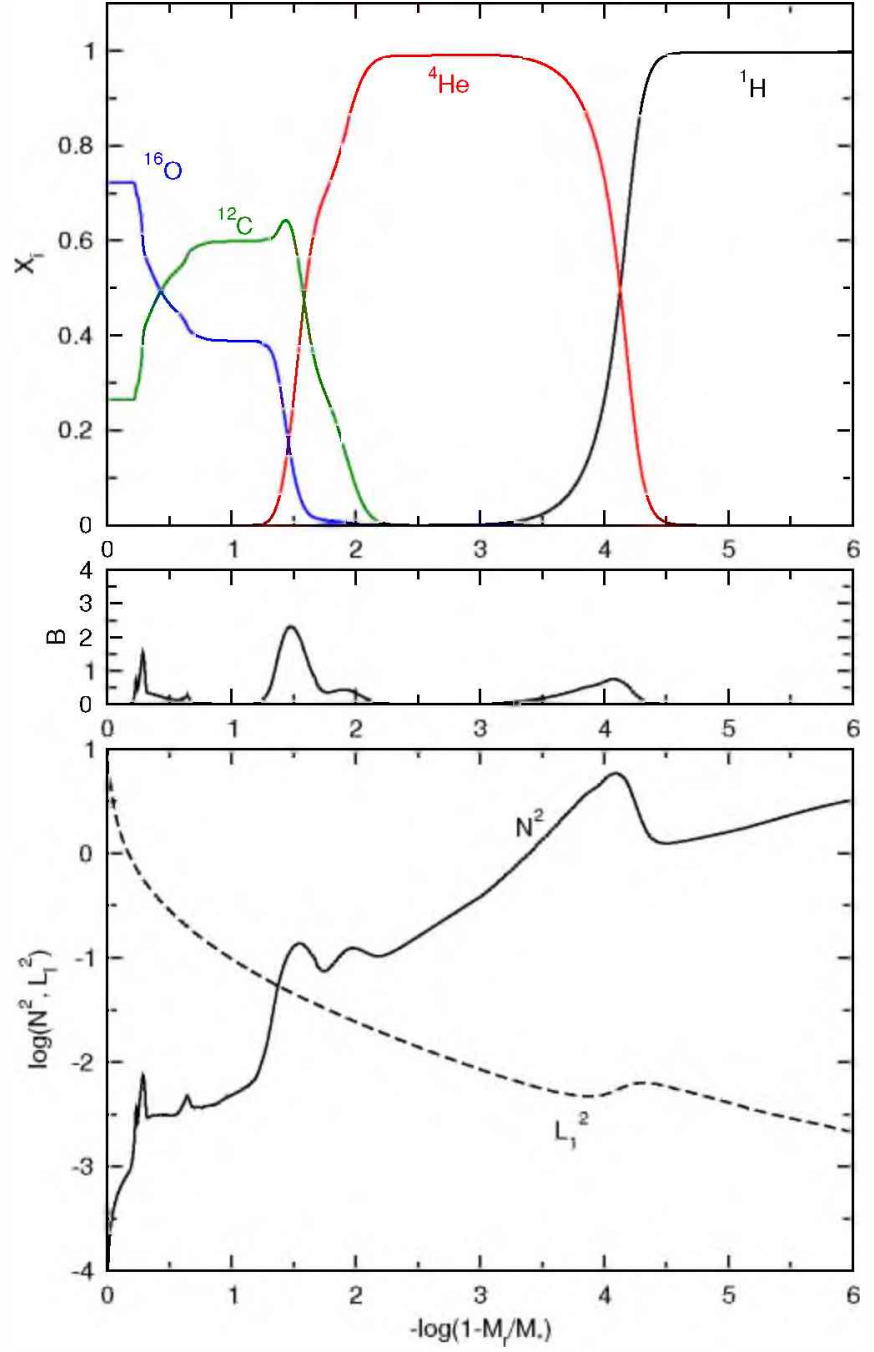

Figure 6. Internal chemical profile (upper pane1), the Ledoux term $B$ (middle panel), and the logarithm of the squared Brunt-Vaisala $(N)$ and Lamb $\left(L_{\ell}\right)$ frequencies (lower panel) in terms of the outer mass fraction $(-\log q$, where $q \equiv 1-M_{r} / M_{*}$ ) for dipole modes $(\ell=1$ ) corresponding to a DA white dwarf model with $M_{*}=0.6096 M_{\odot}$ and $T_{\text {eff }} \sim 12,000 \mathrm{~K}$.

(A color version of this figure is available in the online journal.)

exploration of the parameter space regarding the shape of the chemical abundance profiles in the core. The parameters are the central oxygen abundance $\left(X_{O}\right)$, the coordinate at which $X_{O}$ starts to drop, and the coordinate at which $X_{\mathrm{O}}$ drops to zero - see, e.g., Bischoff-Kim et al. (2008). The overall shape of the core chemical profiles displayed in Figure 7 is substantially simpler than that of the chemical profiles characterizing the model depicted in Figure 6. Note the presence of a chemical transition region of $\mathrm{C}$ and $\mathrm{He}$. This is at variance with the chemical profiles produced by LPCODE, which are characterized by a triple transition region of oxygen, carbon, and helium (see Figure 6). The shape of the $\mathrm{C} / \mathrm{He}$ chemical interface in this model is set by diffusion parameters, which were chosen to match the pulsation periods of G 117-B15A (Bischoff-Kim et al. 2008). It is worth noting that this transition region produces the most prominent bump in the Brunt-Vaisäla frequency (see the Ledoux term $B$ in the central panel of Figure 7). We mention that the presence of a thick pure carbon buffer like that assumed in these models is not expected from stellar evolution calculations. Finally, the He/H chemical transition region has been obtained
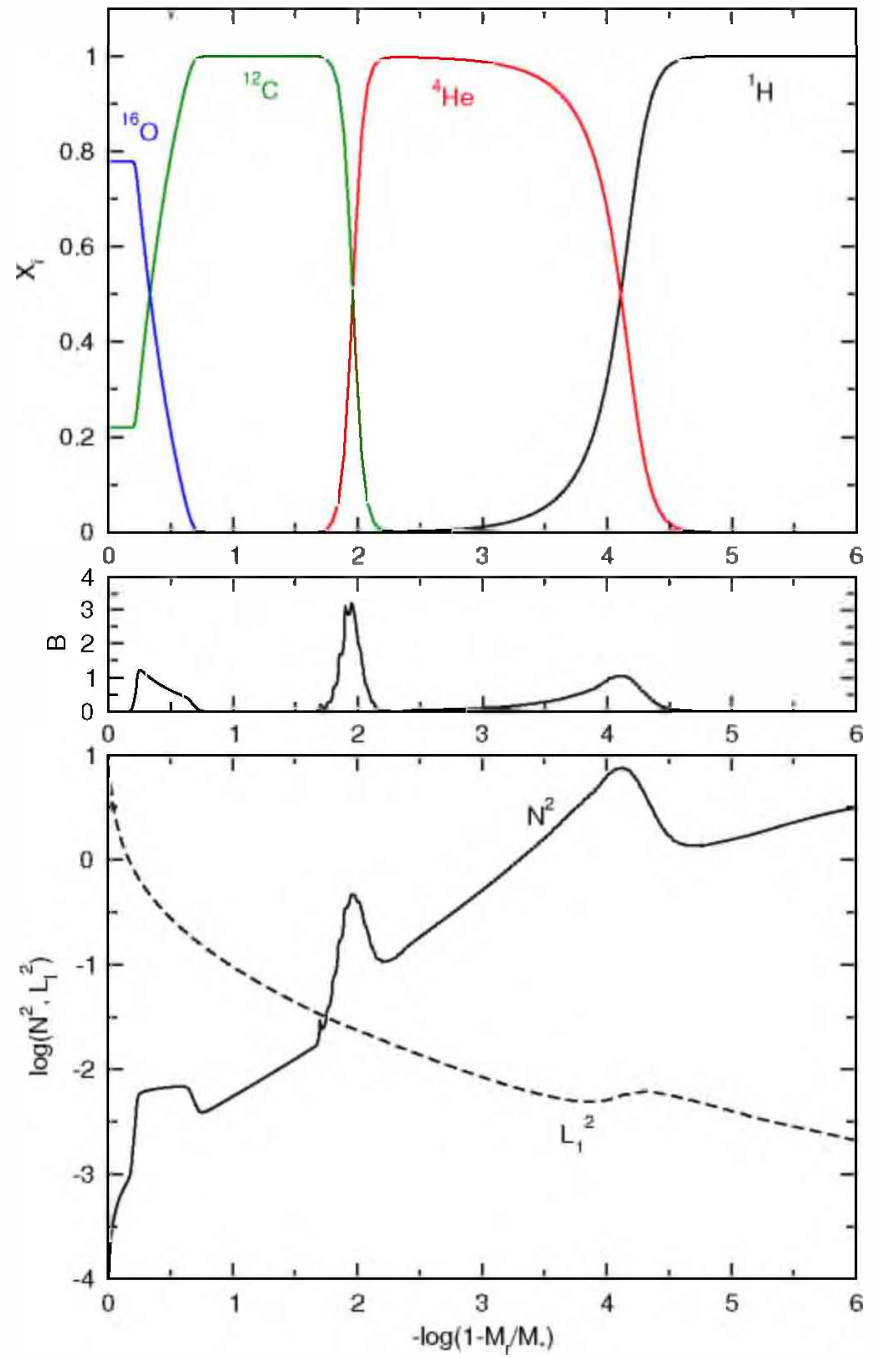

Figure 7. Same as in Figure 6, but for the case of ramp-like core chemical profiles.

(A color version of this figure is available in the online journal.)

by assuming equilibrium diffusion (Arcoragi \& Fontaine 1980), but without the trace element approximation-see BischoffKim et al. (2008) for details. The shape of this interface is very similar to one based on time-dependent diffusion. It is worth noting, however, that the slight differences in the thickness and steepness of this chemical interface between the LPCODE model and the ramp-like model lead to a non-negligible contribution to the differences found in the period spacing (and the period themselves) of the models (see later in this section).

In Figure 8 , we display the case of a template model characterized by a Salaris-like core chemical structure. This kind of core chemical profiles was employed first by Córsico et al. (2001, 2002b) and Benvenuto et al. (2002a, 2002b), and more recently in Bischoff-Kim et al. (2008). Actually, the core chemical profiles displayed in Figure 8 and those used in Bischoff-Kim et al. (2008) are a close adaptation of the original chemical profiles of Salaris et al. (1997). Except for the core region, the rest of the chemical profiles in this model are the same as in the model depicted in Figure 7. At variance with the template models described earlier, in this case there are two core chemical interfaces of oxygen and carbon instead of just one. This leads to four bumps in the Brunt-Väisälä frequency, as can be seen in the lower panel in Figure 8 . 

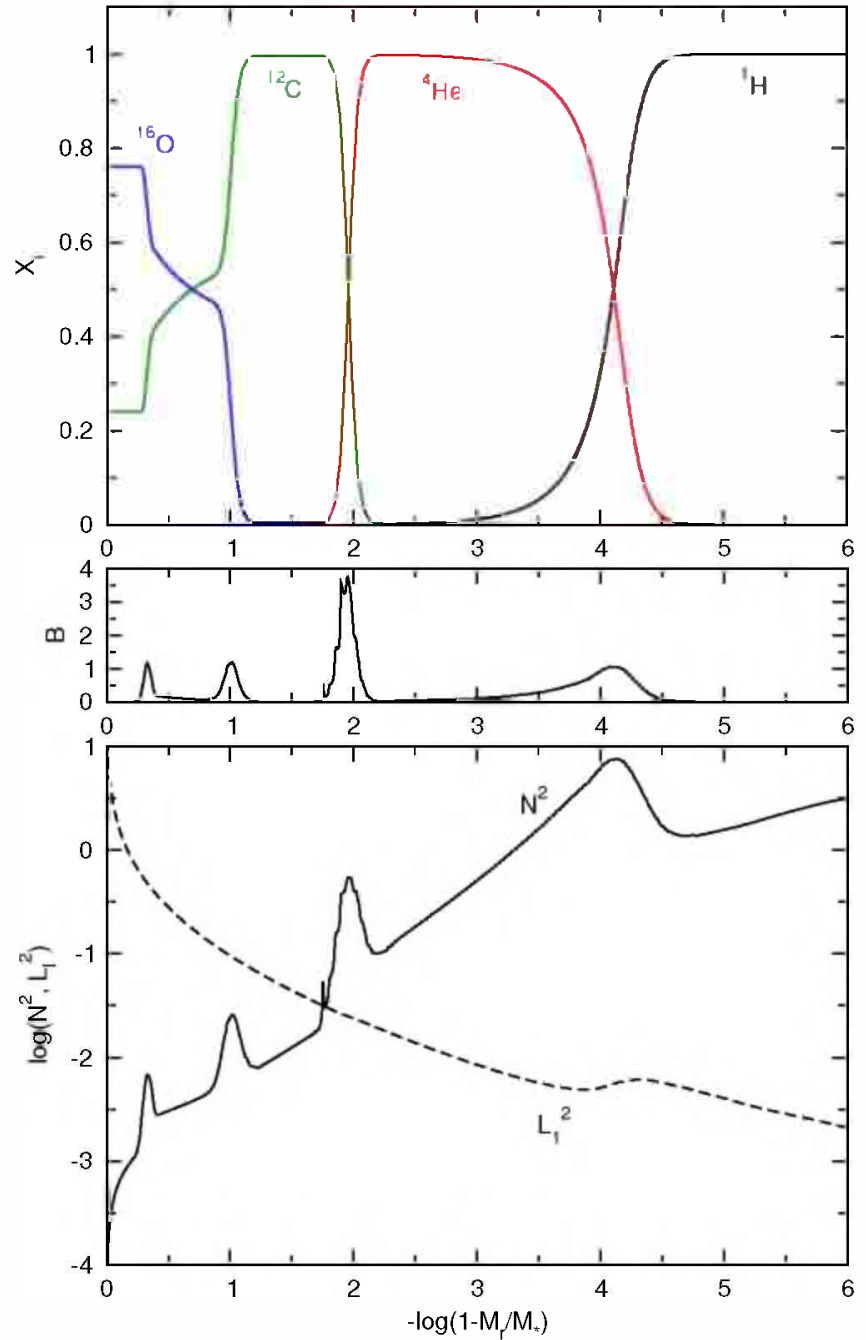

Figure 8. Same as in Figure 6, but for the case of Salaris-like core chemical profiles.

(A color version of this figure is available in the online journal.)

Figures 6- 8 clearly reveal the profound differences existing between the chemical profiles and the run of the Brunt-Väisälä frequency of the model generated with LPCODE, i.e., by considering the full evolution of progenitor stars, and the two template models that incorporate the most widely used chemical profiles in past and current asteroseismological studies of ZZ Ceti stars. The differences are particularly noteworthy in the core chemical structure.

In what follows, we compare the pulsation properties of our template model with the models having core ramp-like and Salaris-like chemical profiles. The $\ell=1$ asymptotic period spacing is largest for the LPCODE model $(45.38 \mathrm{~s})$, followed by the Salaris-like core chemical profile model (44.17 s) and by the ramp-like model (43.32 s). Since these models have the same stellar mass and effective temperature, the period and asymptotic period spacing differences are exclusively due to the differences in the chemical profiles at the core and the envelope of the three models. In particular, the subtle differences existing in the shape of the $\mathrm{He} / \mathrm{H}$ interface resulting from time-dependent diffusion and that obtained from equilibrium diffusion give rise to a nonnegligible contribution to the difference in the asymptotic period spacing.

If we now turn the argument around and imagine doing asteroseismological fits where we fix the chemical profiles to
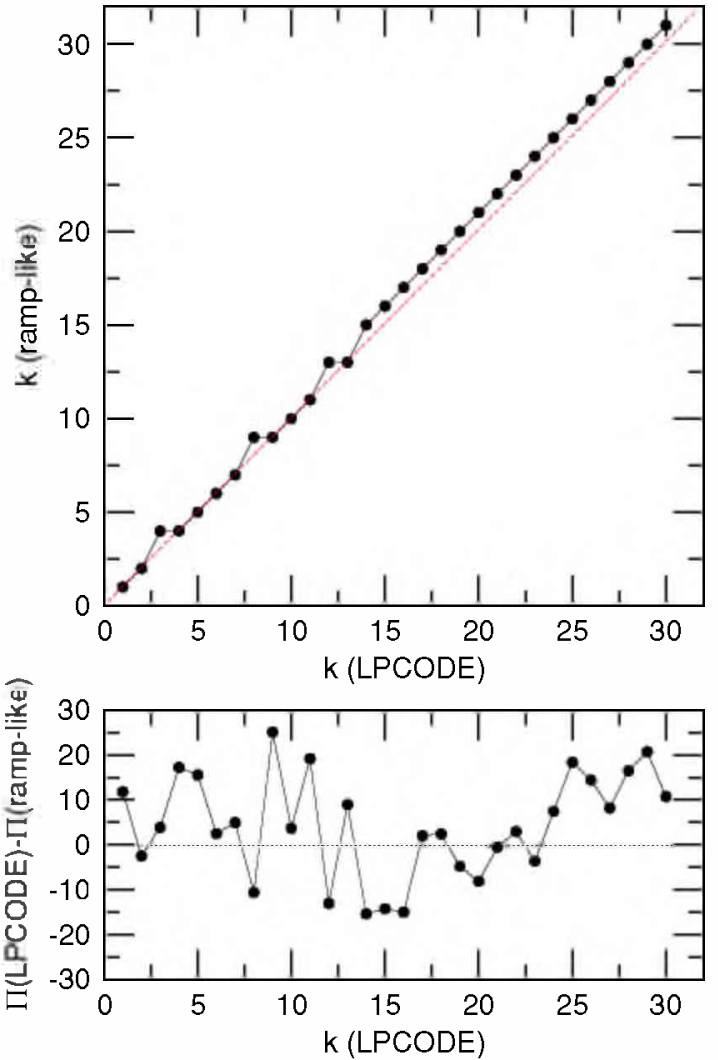

Figure 9. Comparison between the template model generated with LPCODE (Figure 6) and the template model with ramp-like core chemical profiles (Figure 7). The upper panel shows the differences in the $k$ identification, and the lower panel depicts the differences between the matched periods.

(A color version of this figure is available in the online journal.)

those found using LPCODE and allow the mass and effective temperature of the models to vary to give us a pulsation spectrum that matches that of an observed pulsating white dwarf, the asymptotic period spacing of the models will influence the mass and effective temperature of the best-fits models. As it is well known, the hotter and more massive models have smaller asymptotic period spacings (for modes that are not strongly trapped). Since the asymptotic period spacing is larger for the LPCODE models, the best-fit models would have to have larger mass and effective temperature to match a given observed asymptotic period spacing. As a result, we would expect to find asteroseismological fits that are more massive and hotter than current fits. This effect should mainly be observed for rich white dwarf pulsators, where we have a wealth of higher $k$ (asymptotically spaced) modes to fit. For G 117-B15A, for instance, we cannot draw any conclusions from the asymptotic period spacings alone, as the only three modes observed have low radial overtone and are strongly trapped (Bischoff-Kim et al. 2008).

The higher period spacing for the LPCODE model leads to a drift to longer and longer periods as we work down the list of periods toward higher $k$-modes. For instance, the cumulated effect of the $2.06 \mathrm{~s}$ difference between the LPCODE and the ramplike models results in higher $k$ periods to differ by as much as $65 \mathrm{~s}$ (for $k \sim 30$ if $\ell=1$ and $k \sim 55$ if $\ell=2$ ). The practical result in asteroseismological studies of such a drift to higher periods would be to lead to different $k$ identifications of modes. In Figures 9 and 10, we show how asteroseismological fits may be affected. In the upper panels we show the differences in $k$ 

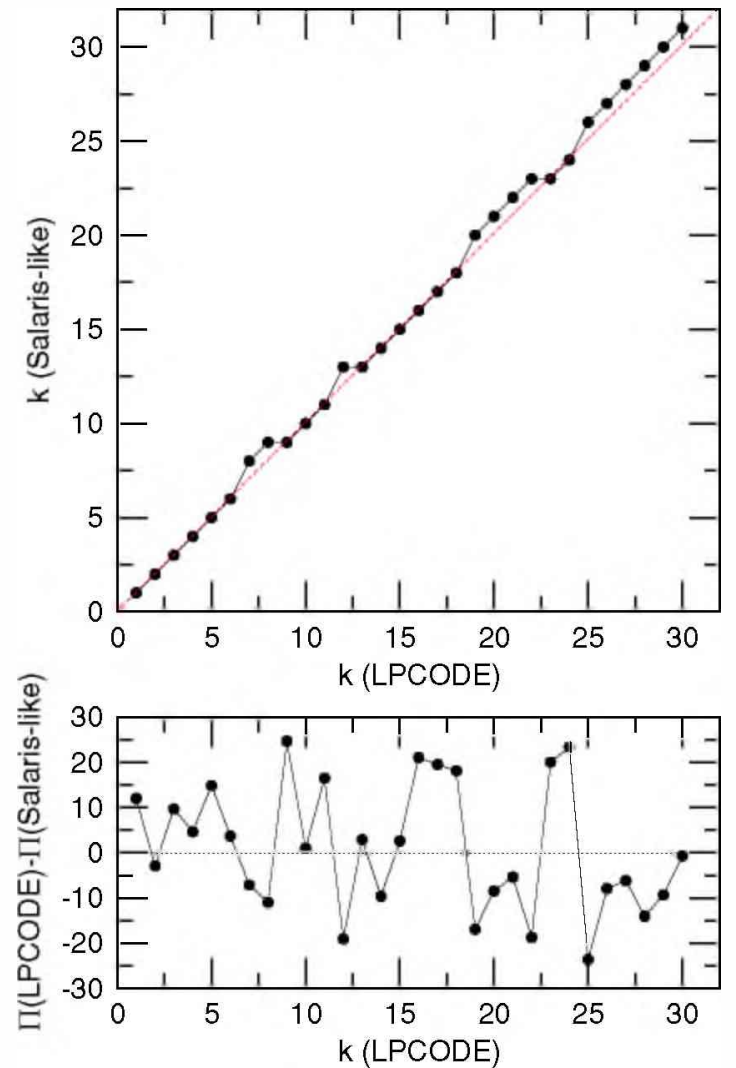

Figure 10. Same as in Figure 9, but for the comparison between the template model generated with LPCODE (Figure 6) and the template model with Salarislike core chemical profiles (Figure 8).

(A color version of this figure is available in the online journal.)

identifications, and in the bottom panels we show how the period of the matched modes differ. Even allowing the $k$ identifications to change to find the best match between the periods of the

two models compared, we still find that some individual periods differ by as much as $\sim 25 \mathrm{~s}$.

We conclude our analysis by examining the forward period spacing $\left(\Delta \Pi_{k} \equiv \Pi_{k+1}-\Pi_{k}\right)$ and the kinetic energy $\left(E_{\mathrm{kin}}\right)$ of the three template models (Figure 11). The kinetic energy is computed according to Córsico \& Althaus (2006). The horizontal lines in red correspond to the asymptotic period spacing. As is well known, the presence of density gradients in the chemical transition regions in the interior of a white dwarf causes mode trapping (Winget et al. 1981; Brassard et al. 1992a; Córsico et al. 2002a). The signature of mode trapping on the period-spacing distribution is the presence of strong minima in a $\Delta \Pi_{k}-\Pi_{k}$ diagram, in contrast to the situation in which the star is chemically homogeneous-see, for instance, Córsico \& Benvenuto (2002). Due to the presence of several chemical interfaces in the template models, we expect to find clear signatures of mode trapping.

For the model generated with LPCODE, the $\Delta \Pi_{k}$ distribution exhibits two primary minima and several secondary minima, as can be seen in the upper left panel of Figure 11. In the case of the model with ramp-like core chemical profiles (upper central panel) there are also primary and secondary minima, but the contrast amongst them is much less pronounced than in the case of the LPCODE model. Finally, in the case of the model with Salaris-like core chemical profiles (upper right panel) there is no clear distinction between primary and secondary minima of $\Delta \Pi_{k}$. That is, all the minima are very similar.

In spite of the complexity of the mode-trapping pattern exhibited by the template models, it is possible to make some rough inferences by examining the values of the kinetic energy of the modes (lower panels of Figure 11). A close inspection of the plots reveals that each minimum of $\Delta \Pi_{k}$ is associated with a maximum in $E_{\text {kin }}$ of a mode with the same radial order $k$ or differing in $\Delta k= \pm 1$. Modes corresponding to local maxima in $E_{\text {kin }}$ are modes partially confined to the core regions below the $\mathrm{O} / \mathrm{C}$ and/or the $\mathrm{C} / \mathrm{He}$ chemical interfaces (the $\mathrm{O} / \mathrm{C} / \mathrm{He}$
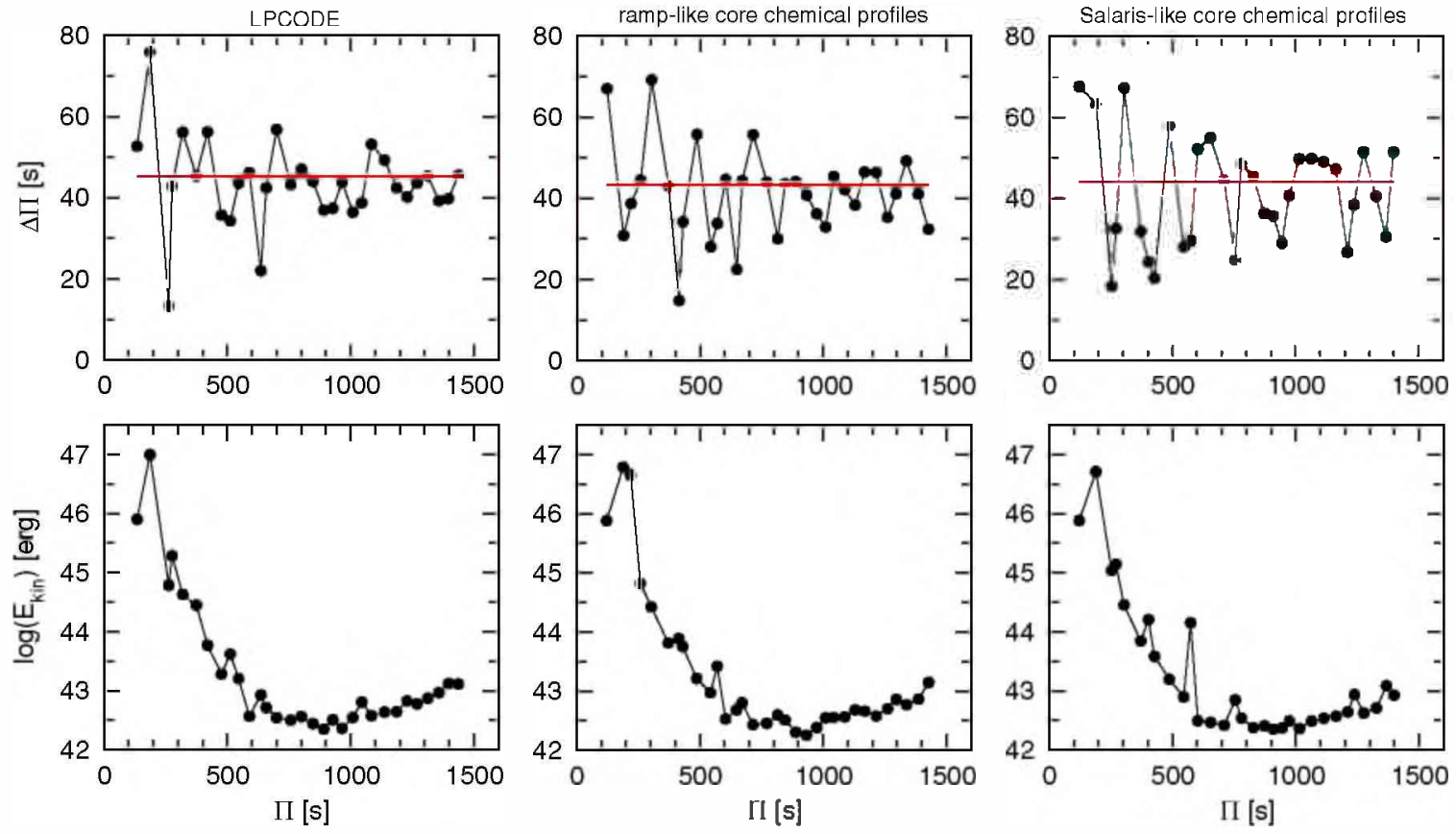

Figure 11. Upper panels show the forward period spacing and the lower panels depict the logarithm of the oscillation kinetic energy of $\ell=1$ modes in terms of the pulsation periods. The horizontal lines in the upper panels display the asymptotic period spacing.

(A color version of this figure is available in the online journal.) 
transition in the case of the LPCODE model), that is, modes with amplitudes relatively large even in very deep layers of the model. These modes are potentially useful from an asteroseismological point of view. The remaining modes (which have neither maxima nor minima kinetic energy values) are much less sensitive to the presence of the chemical transition regions, and so, they are of minor relevance for asteroseismolgy.

We conclude that the pulsation properties of DA white dwarf models that incorporate our new chemical profiles for the core and envelope substantially differ from those of models having the most commonly used chemical profiles. The important issue to be addressed now is to asses the impact of our new chemical profiles on asteroseismological period-to-period fits of real DAV stars. This step is beyond the scope of the present work, and we defer it to future papers.

\section{SUMMARY AND CONCLUSIONS}

In this paper, we computed new chemical profiles for the core and envelope of white dwarfs appropriate for pulsational studies of ZZ Ceti stars. These profiles were derived from the full and complete evolution of progenitor stars from the ZAMS, through the thermally pulsing and mass-loss phases on the AGB. These new profiles are intended for asteroseismological studies of ZZ Ceti stars that require realistic chemical profiles throughout the white dwarf interiors. In deriving the new chemical profiles, we employed the LPCODE evolutionary code, based on detailed and updated constitutive physics. Extramixing episodes during central hydrogen and helium burning, time-dependent element diffusion during the white dwarf stage, and chemical rehomogenization of the inner carbon-oxygen composition by RT instabilities were considered. The metallicity of progenitor stars is $Z=0.01$.

We discussed at some length the importance of the initial-final mass relationship for the white dwarf carbonoxygen composition. A reduction of the efficiency of extramixing episodes during the thermally pulsing AGB phase, supported by different pieces of theoretical and observational evidence, yields a gradual increase of the hydrogen-free core mass as evolution proceeds during this phase. As a result, the initial-final mass relationship by the end of the thermally pulsing $\mathrm{AGB}$ is markedly different from that resulting from considering the mass of the hydrogen free core right before the first thermal pulse. We found that this issue has implications for the carbon-oxygen composition expected in a white dwarf. In particular, the central oxygen abundance may be underestimated by about $15 \%$ if we assume the white dwarf mass to be the hydrogen-free core mass before the first thermal pulse. We also discuss the importance of the computation of the thermally pulsing AGB and element diffusion for the chemical profiles expected in the outermost layers of ZZ Ceti stars. In this sense, we found a strong dependence of the outer layer chemical stratification on the stellar mass. In less massive models, the intershell region rich in helium and carbon-which is built during the mixing episode at the last AGB thermal pulse-is not removed by diffusion by the time the $\mathrm{ZZ}$ Ceti stage is reached.

Finally, we performed adiabatic pulsation computations and discussed the implications of our new chemical profiles for the pulsational properties of $\mathrm{ZZ}$ Ceti stars. We found that the whole $g$-mode period spectrum and the mode-trapping properties of these pulsating white dwarfs as derived from our new chemical profiles are substantially different from those based on the most widely used chemical profiles in existing asteroseismological studies.
We expect the best-fit parameters of asteroseismological studies using the LPCODE chemical profiles to differ significantly from those found in studies made so far; further studies will show in what way. Will we solve the high-mass problem with G117-B15A and Salaris-like core profiles (Bischoff-Kim et al. 2008 ) or find thicker hydrogen layers in asteroseismological fits, more in line with stellar evolution calculations (Castanheira \& Kepler 2008)?

Part of this work was supported by AGENCIA through the Programa de Modernización Tecnológica BID 1728/OC-AR, by the PIP 112-200801-00940 grant from CONICET, by the AGAUR, by MCINN grant AYA2008-04211-C02-01, and by the European Union FEDER funds. This research has made use of NASA's Astrophysics Data System.

\section{REFERENCES}

Alexander. D. R., \& Ferguson. J. W. 1994. ApJ, 437. 879

Althaus, L. G., \& Benvenuto, O. G. 2000, MNRAS, 317,952

Althaus, L. G., García-Berro, E., Isern, J., Córsico, A. H., \& Rohrmann, R. D. 2007, A\&A, 465, 249

Althaus, L. G., Panei. J. A., Miller Bertolami. M. M., García-Berro. E., Córsico. A. H., Romero, A. D., Kepler, S. O., \& Rohrmann, R. D. 2009a, ApJ, 704. 1605

Althaus, L. G.. Panei. J. A., Romero, A. D., Rohrmann. R. D., Córsico, A. H. García-Berro, E.. \& Miller Bertolami, M. M. 2009b. A\&A. 502, 207

Althaus, L.G., Serenelli, A. M., Córsico, A. H., \& Montgomery, M. H. 2003, A\&A, 404, 593

Althaus. L. G., et al. 2005. A\&A, 435.631

Angulo, C., et al. 1999, Nucl. Phys. A, 656, 3

Arcoragi, J.-P.. \& Fontaine, G. 1980, ApJ, 242, 1208

Benvenuto. O. G.. Córsico, A. H.. Althaus. L. G.. \& Serenelli. A. M. 2002a, MNRAS, 332, 399

Benvenuto, O. G., Córsico, A. H., Althaus, L. G., \& Serenelli, A. M. 2002b, MNRAS, 335, 480

Bischoff-Kim. A. 2009. AIP Conf. Ser. 1170. Stellar Pulsation: Challenges for Theory and Observation. ed. J. A. Guzik \& P. A. Bradley (Melville. NY: AIP), 621

Bischoff-Kim. A., Montgomery, M. H., \& Winget, D. E. 2008, ApJ, 675. 1505

Bognár, Z.. Paparó, M.. Bradley, P. A.. \& Bischoff-Kim. A. 2009. MNRAS. 399, 1954

Bradley, P. A. 1996, ApJ, 468, 350

Bradley, P. A. 1998, ApJS, 116, 307

Bradley, P. A. 2006, Mem. Soc. Astron. Ital., 77, 437

Bradley, P. A. 2001, ApJ, 552, 326

Bradley. P.. \& Kleinman. S. J. 1997. in White Dwarfs, ed. J. Isern. M. Hernanz. \& E. García-Berro (Astrophys. Space Sci. Lib. Vol. 214; Dordrecht: Kluwer). 445

Brassard, P., Fontaine, G., Wesemael, F., \& Hansen, C. J. 1992a, ApJS, 80, 369 Brassard, P., Fontaine, G., Wesemael, F., Kawaler, S. D., \& Tassoul, M. 1991. ApJ, 367, 601

Brassard, P., Fontaine, G., Wesemael, F., \& Tassoul, M. 1992b, ApJS, 81, 747

Brickhill, A. J. 1991, MNRAS, 251, 673

Burgers, J. M. 1969. Flow Equations for Composite Gases (New York: Academic)

Cassisi, S., Potekhin, A. Y.. Pietrinferni, A., Catelan, M., \& Salaris, M. 2007, ApJ, 661, 1094

Castanheira, B. G., \& Kepler, S. O. 2008, MNRAS, 385, 430

Castanheira, B. G., \& Kepler, S. O. 2009, MNRAS, 396, 1709

Catalán, S., Isern, J., García-Berro, E.. \& Ribas. I. 2008, MNRAS, 387, 1693

Caughlan, G. R., Fowler. W. A., Harris, M. J., \& Zimmermann. B. A. 1985. At. Data Nucl. Data Tables, 32, 197

Córsico, A. H., \& Althaus, L. G. 2006, A\&A, 454, 863

Córsico, A. H., Althaus, L. G., Benvenuto, O. G., \& Serenelli, A. M. 2002a. A\&A. 387. 531

Córsico, A. H., Althaus, L. G., Montgomery, M. H., García-Berro, E., \& Isern. J. 2005, A\&A, 429, 277

Córsico, A. H.. \& Benvenuto, O. G. 2002. Ap\&SS. 279. 281

Córsico, A. H., Benvenuto, O. G., Althaus, L. G., Isern, J., \& García-Berro, E 2001, New Astron., 6, 197

Córsico, A. H., Benvenuto, O. G., Althaus, L. G., \& Serenelli, A. M. 2002b. MNRAS. 332, 392 
Cörsico, A. H., García-Berro, E., Althaus, L. G., \& Isern, J. 2004. A\&A, 427. 923

Dolez, N., \& Vauclair, G. 1981, A\&A, 102, 375

Dziembowski, W., \& Koester, D. 1981, A\&A, 97, 16

Fontaine, G., \& Brassard. P. 2008, PASP, 120, 1043

Fontaine. G., Brassard. P., Bergeron. P.. \& Wesemael, F. 1992. ApJ. 399 L91

Goldreich. P., \& Wu, Y. 1999, ApJ, 511, 904

Grevesse, N., \& Sauval, A. J. 1998, Space Sci. Rev, 85, 161

Haft, M., Raffelt, G., \& Weiss, A. 1994. ApJ, 425, 222

Herwig. F., Blocker. T., Schonberner. D.. \& El Eid. M. 1997. A\&A. 324. L 81

Iglesias, C. A., \& Rogers, F. J. 1996, ApJ, 464, 943

Isern. J., García-Berro, E., Althaus, L. G., \& Córsico, A. H. 2010, A\&A, 512. A86

Isern. J., García-Berro, E.. Hernanz, M., \& Chabrier, G. 2000, ApJ, 528. 397

Isern, J., Hernanz, M., \& García-Berro, E. 1992, ApJ, 392, L23

Itoh, N., Hayashi, H., Nishikawa, A., \& Kohyama, Y. 1996, ApJS, 102, 411

Kanaan, A.. et al. 2005, A\&A. 432. 219

Landolt. A. U. 1968, ApJ. 153, 151

Lugaro, M., Herwig, F., Lattanzio, J. C., Gallino, R., \& Straniero, O. 2003, ApJ 586,1305

Marigo, P.. \& Aringer, B. 2009, A\&A, 508, 1539

Metcalfe, T. S. 2003, ApJ, 587, L43

Metcalfe, T. S., Montgomery, M. H., \& Kanaan. A. 2004, ApJ. 605. L133

Metcalfe, T. S., Montgomery, M. H., \& Kawaler. S. D. 2003. MNRAS. 344 L88

Miller Bertolami, M. M., \& Althaus, L. G. 2006, A\&A, 454, 845
Miller Bertolami, M. M., Althaus, L. G., Unglaub, K.. \& Weiss, A. 2008, A\&A. 491,253

Montgomery, M. H., Klumpe, E. W., Winget, D. E., \& Wood, M. A. 1999, ApJ, 525,482

Mullally, F., Winget, D. E., De Gennaro, S., Jeffery, E., Thompson, S. E., Chandler. D.. \& Kepler, S. O. 2008. ApJ, 676, 573

Pech. D., \& Vauclair. G. 2006. A\&A. 453, 219

Pech, D., Vauclair, G., \& Dolez, N. 2006, A\&A, 446, 223

Pfeiffer, B., et al. 1996, A\&A, 314, 182

Renedo, I., Althaus, L. G., Miller Bertolami, M. M., Romero, A. D. Córsico, A. H., Rohrmann. R. D.. \& García-Berro, E. 2010. ApJ, in press (arXiv:1005.2170)

Salaris, M., Dominguez, I., García-Berro, E., Hernanz, M., Isern, J., \& Mochkovitch, R. 1997, ApJ, 486, 413

Salaris, M., Serenelli, A., Weiss, A., \& Miller Bertolami, M. 2009, ApJ, 692. 1013

Schroder, K. P., \& Cuntz, M. 2005, ApJ, 630, L73

Segretain, L., Chabrier, G., Hernanz, M., García-Berro, E., Isern, J., \& Mochkovitch, R. 1994. ApJ, 434, 641

Straniero, O.. Domínguez. I.. Imbriani. G.. \& Piersanti. L. 2003. ApJ. 583, 878

Tassoul. M.. Fontaine. G.. \& Winget, D. E. 1990. ApJS, 72, 335

Unglaub, K., \& Bues, I. 2000, A\&A, 359, 1042

Vassiliadis, E., \& Wood, P. R. 1993, ApJ, 413, 641

Weiss, A., \& Ferguson, J. W. 2009, A\&A, 508, 1343

Winget. D. E., \& Kepler. S. O. 2008. ARA\&A. 46, 157

Winget. D. E., van Horn. H. M., \& Hansen. C. J. 1981. ApJ, 245. L33

Winget, D. E., van Horn, H. M., Tassoul, M., Fontaine, G., Hansen, C. J., \& Carroll, B. W. 1982, ApJ, 252, L65 